\title{
Performance of Digital Linear Modulations on Weibull Slow-Fading Channels
}

\author{
Julian Cheng, Member, IEEE, Chinthananda Tellambura, Senior Member, IEEE, and Norman C. Beaulieu, Fellow, IEEE
}

\begin{abstract}
A closed-form expression is derived for the moment-generating function of the Weibull distribution, valid when its fading parameter assumes integer values. Expressions for average signal-to-noise ratio, signal outage, and average symbol-error rate are derived for single-channel reception and independent multichannel diversity reception operating on flat Weibull slow-fading channels.
\end{abstract}

Index Terms-Error analysis, fading channels, Laplace transforms, Weibull distributions.

\section{INTRODUCTION}

$\mathbf{T}$ HE Weibull distribution is a flexible statistical model for describing multipath fading channels for both indoor and outdoor propagation environments. Experimental data supporting the Weibull fading model was reported by Shepherd [1], and Hashemi considered its use as a model for indoor fading channels in [2]. Based on fading-channel data obtained from a recent measurement program at $900 \mathrm{MHz}$, Tzeremes and Christodoulou also reported that the Weibull distribution can be used to model outdoor fading well in some cases [3]. The IEEE Vehicular Technology Society Committee on Radio Propagation also recommended the use of a Weibull or a Nakagami model for theoretical studies to introduce slope changes in the tail of the model distribution, to compensate some shortcomings of the Rayleigh distribution [4]. A large body of literature has been devoted to the study of digital communications over Nakagami channels. However, with the exception of [5] and [6], there exist few results pertaining to transmission over Weibull fading channels.

The moment-generating function (MGF) method is gaining popularity as an approach to unified error-rate analysis of digital communications over fading channels [7]. Both average symbol-error rates (ASERs) and outage probabilities (OPs) can be expressed in terms of the MGF of the signal-to-noise ratio (SNR) in a single-channel receiver system, and the MGF of the SNR at the output of a diversity combiner in a multichannel receiver system. A tractable and useful expression for the MGF of the Weibull distribution is not known. A closed-form expression for the Weibull MGF is derived in this letter, and used with the MGF method for a unified performance evaluation. A power-series expansion is known for the MGF, but the series does not always converge. Further, the power series may converge theoretically, but not in practical computation, because the terms oscillate between very large positive and negative

Paper approved by M.-S. Alouini, the Editor for Modulation and Diversity Systems of the IEEE Communications Society. Manuscript received July 31, 2003; revised November 21, 2003 and January 19, 2004. This paper was presented in part at the IEEE Vehicular Technology Conference, Orlando, FL, 2003.

The authors are with the Department of Electrical and Computer Engineering, University of Alberta, Edmonton, AB T6G 2V4, Canada (e-mail: beaulieu@ee.ualberta.ca).

Digital Object Identifier 10.1109/TCOMM.2004.833015 values before becoming ultimately decreasing. In this letter, we derive a closed-form expression for the MGF of a Weibull distribution in terms of a known hypergeometric function. Using this MGF expression, we study the outage and symbol-error rate (SER) performances (for uncoded systems) of some important linear modulation schemes in both single-channel reception and independent multichannel diversity reception on flat Weibull slow-fading channels.

\section{WEIBULL MGF}

The probability density function (PDF) of the Weibull distribution is

$$
f_{X}(x)=\frac{c x^{c-1}}{\beta} \exp \left(-\frac{x^{c}}{\beta}\right), \quad c>0, \quad x \geq 0
$$

where the index $c$ is called the Weibull fading parameter and $\beta$ is a positive scale parameter. The root-mean-square (rms) value of the Weibull fading amplitude is $\beta^{1 / c}[(2 / c) \Gamma(2 / c)]^{1 / 2}$, where $\Gamma(\cdot)$ is the Gamma function. The Weibull fading parameter can take values between 0 and $\infty$. In the special case when $c=1$, the Weibull distribution becomes an exponential distribution; when $c=2$, the Weibull distribution specializes to a Rayleigh distribution.

The $\mathrm{MGF}^{1}$ of the Weibull distribution is

$$
\begin{aligned}
\mathcal{M}_{X}(s, c) & =\int_{0}^{\infty} e^{-s x} f_{X}(x) d x \\
& =\frac{c}{\beta} \int_{0}^{\infty} \exp \left(-s x-\frac{x^{c}}{\beta}\right) x^{c-1} d x
\end{aligned}
$$

where $\mathcal{M}_{X}(s, c)$ is used to explicitly denote the dependence of the MGF on $c$. In this letter, we assume that $c$ takes only integer values and this condition is required for our mathematical solution. Then approximate results for OP or ASER for noninteger values of $c$ can be obtained using interpolation of the final results obtained for integer values. We present a closed-form expression for the MGF of the Weibull distribution in terms of a confluent hypergeometric function. To show this, we define an integral $^{2} I$ as

$$
I=\int_{0}^{\infty} x^{p-1} e^{-z x-\alpha x^{r}} d x, \quad \alpha, z, p>0
$$

\footnotetext{
${ }^{1}$ In this letter, the definition of the MGF differs from the conventional definition by a negative exponent, and is essentially the Laplace transform of a PDF.

${ }^{2}$ The integral (3) is called Faxen's integral [8]. Bakhoom first suggested that Faxen's integral with integer $r$ values can be expressed in terms of the generalized hypergeometric function [9]. However, he did not provide a solution similar to ours.
} 
where $r$ is a positive integer. In the Appendix, using a Mellin's transform approach, we show that

$$
I=(2 \pi)^{\frac{1-r}{2}} r^{p-\frac{1}{2}} z^{-p} G_{1, r}^{r, 1}\left(\frac{z^{r}}{\alpha r^{r}} \mid \frac{p}{r}, \frac{(p+1)}{r}, \ldots, \frac{1}{r}\right)
$$

where $G_{p, q}^{m, n}(\cdot)$ is the Meijer's $G$ function [10, eq. (9.301)]. The Meijer's $G$ function is widely available in many scientific software packages, such as Mathematica and Maple. Applying (4) to (2), we obtain the MGF for the Weibull distribution as

$$
\begin{aligned}
& \mathcal{M}_{X}(s, c)=\left(\frac{c}{\beta}\right)(2 \pi)^{\frac{1-c}{2}} c^{\left(c-\frac{1}{2}\right)} s^{-c} \\
& \times G_{1, c}^{c, 1}\left(\beta\left(\frac{s}{c}\right)^{c} \mid \begin{array}{c}
1 \\
\left.1,1+\frac{1}{c}, \ldots, 1+\frac{(c-1)}{c}\right) .
\end{array}\right.
\end{aligned}
$$

Two special cases of the Weibull distribution can be used to test the correctness of (5). When $c=1$, from (5), the MGF becomes

$$
\mathcal{M}_{X}(s, 1)=\frac{1}{\beta s} G_{1,1}^{1,1}\left(\beta s \mid \begin{array}{l}
1 \\
1
\end{array}\right) .
$$

It is known that [11]

$$
G_{1,1}^{1,1}\left(\begin{array}{l|l}
a z & \begin{array}{l}
a_{1} \\
b_{1}
\end{array}
\end{array}\right)=\frac{a^{b_{1}} \Gamma\left(1+b_{1}-a_{1}\right) z^{b_{1}}}{(1+a z)^{1+b_{1}-a_{1}}} .
$$

From (6) and (7), we obtain $\mathcal{M}_{X}(s, 1)=1 /(1+\beta s)$, which is the Laplace transform of the exponential PDF, as expected. When $c=2$, from (5), the MGF becomes

$\mathcal{M}_{X}(s, 2)=\left(\frac{2}{\beta}\right)(2 \pi)^{-\frac{1}{2}}\left(2^{\frac{3}{2}}\right) s^{-2} G_{1,2}^{2,1}\left(\frac{\beta s^{2}}{4} \mid \begin{array}{c}1 \\ 1,1+\frac{1}{2}\end{array}\right)$.

It is known that [11]

$$
\begin{aligned}
G_{1,2}^{2,1}\left(z \mid \begin{array}{c}
a_{1} \\
b_{1}, b_{2}
\end{array}\right)=\Gamma\left(b_{1}-a_{1}\right. & +1) \Gamma\left(b_{2}-a_{1}+1\right) z^{\frac{b_{1}+b_{2}-1}{2}} e^{\frac{z}{2}} \\
& \times W_{\frac{2 a_{1}-b_{1}-b_{2}-1}{2}}, \frac{b_{1}-b_{2}}{2}(z)
\end{aligned}
$$

where $W_{k, \mu}(z)$ is the Whittaker function [10]. Applying (9) to (8) and using [10, eqs. (3.381), (8.350), and (8.356)], one can show $\mathcal{M}_{X}(s, 2)=1-\sqrt{\beta \pi} s e^{\left(\beta s^{2} / 4\right)} Q(\sqrt{\beta / 2} s)$ where $Q(x)=\int_{x}^{\infty}(1 / \sqrt{2 \pi}) e^{-t^{2} / 2} d t$, which is the Laplace transform of the Rayleigh PDF, as expected.

It is well known that the $k$ th power of a Weibull-distributed random variable $(\mathrm{RV})$ with parameters $(c, \beta)$ gives another Weibull-distributed $\mathrm{RV}$ with parameters $(c / k, \beta)$. We note here, for use in the following, that if $\mathcal{M}_{X_{i}}(s, c)$ is the MGF of a Weibull RV $X_{i}$, the MGF of $X_{i}^{2}$ is $\mathcal{M}_{X_{i}^{2}}(s, c / 2)$.

\section{Performance Analysis of Digital Modulations on Weibull ChanNels}

\section{A. Single-Channel Reception}

Let $Y$ denote the channel-output SNR for single-channel reception or the combiner-output SNR for multichannel diversity reception. Assuming that noise variance in each channel is unity, the output SNR becomes $Y=X^{2}$, where $X$ is the Weibull fading amplitude. The $k$ th moment of $Y$ is given by

$$
\mathbb{E}\left[Y^{k}\right]=\mathbb{E}\left[X^{2 k}\right]=\beta^{\frac{2 k}{c}} \Gamma\left(1+\frac{2 k}{c}\right) .
$$

In the special case when $k=1$, we have the average output SNR for a single Weibull channel as $\overline{\mathrm{SNR}_{\mathrm{o}}}=\mathbb{E}[Y]=\beta^{2 / c} \Gamma(1+$ $2 / c)$.

The outage probability at outage threshold $\xi$ for singlechannel reception can be calculated as

$$
P_{\text {out }}(\xi)=\operatorname{Pr}(Y<\xi)=1-\exp \left(-\frac{\xi^{\frac{c}{2}}}{\beta}\right) .
$$

Using (5), the ASERs for many digital linear modulations operating on Weibull channels with single-channel reception can be straightforwardly calculated using the MGF method [7]. The details are omitted here, as they are well documented elsewhere.

\section{B. Selection Combining (SC)}

Using the single-channel OP, one can show that the OP for $L$-branch independent $\mathrm{SC}$ is

$$
P_{\text {out }, \mathrm{sc}}(\xi)=\left[1-\exp \left(-\frac{\xi^{\frac{c}{2}}}{\beta}\right)\right]^{L} .
$$

From (12) and [10, eq. (3.381)], one can show the $k$ th moment of SC combiner-output SNR is

$$
\mathbb{E}\left[Y^{k}\right]=L \beta^{\frac{2 k}{c}} \sum_{i=0}^{L-1}(-1)^{i}\left(\begin{array}{c}
L-1 \\
i
\end{array}\right) \frac{\Gamma\left(\frac{2 k}{c}+1\right)}{(i+1)^{\frac{2 k}{c}}+1} .
$$

When $k=1$, we obtain the average output SNR as $\overline{\mathrm{SNR}_{\mathrm{o}, \mathrm{sc}}}=$ $\mathbb{E}[Y]$.

To calculate the ASER using SC reception, it is required to calculate the MGF of $\gamma_{t}$, the SNR at the output of the SC. By using the derivative property of the Laplace transform, one can relate the MGF of a RV to its cumulative distribution function $(\mathrm{CDF})$ as

$$
\mathcal{M}_{Z}(s)=s \int_{0}^{\infty} \exp (-s z) F_{Z}(z) d z .
$$

For our problem, the CDF of the SNR at the SC output can be shown to be

$$
\begin{aligned}
F_{\gamma_{t}}\left(\gamma_{t}\right) & =\left[1-\exp \left(-\frac{\gamma_{t}^{\frac{c}{2}}}{\beta}\right)\right]^{L} \\
& =\sum_{n=0}^{L}(-1)^{n}\left(\begin{array}{l}
L \\
n
\end{array}\right) \exp \left(-\frac{n \gamma_{t}^{\frac{c}{2}}}{\beta}\right) .
\end{aligned}
$$

Using (14) and (15), one can show that the MGF of the SC output SNR is

$$
\begin{aligned}
\mathcal{M}_{\gamma_{t}}(s)=1+ & (2 \pi)^{\frac{1}{2}-\frac{c}{4}} \sqrt{\frac{c}{2}} \sum_{n=1}^{L}\left(\begin{array}{c}
L \\
n
\end{array}\right)(-1)^{n} \\
& \times G_{1, \frac{c}{2}}^{\frac{c}{2}, 1}\left(\left(\frac{\beta}{n}\right)\left(\frac{2 s}{c}\right)^{\frac{c}{2}} \mid \begin{array}{c}
1 \\
\frac{2}{c}, \frac{4}{c}, \ldots, 1
\end{array}\right)
\end{aligned}
$$

where in obtaining (16), we have used the integral identity (4). Using (16) and (5), the ASERs of SC for many digital linear modulations can be readily evaluated via the MGF method [7, Ch. 9]. 


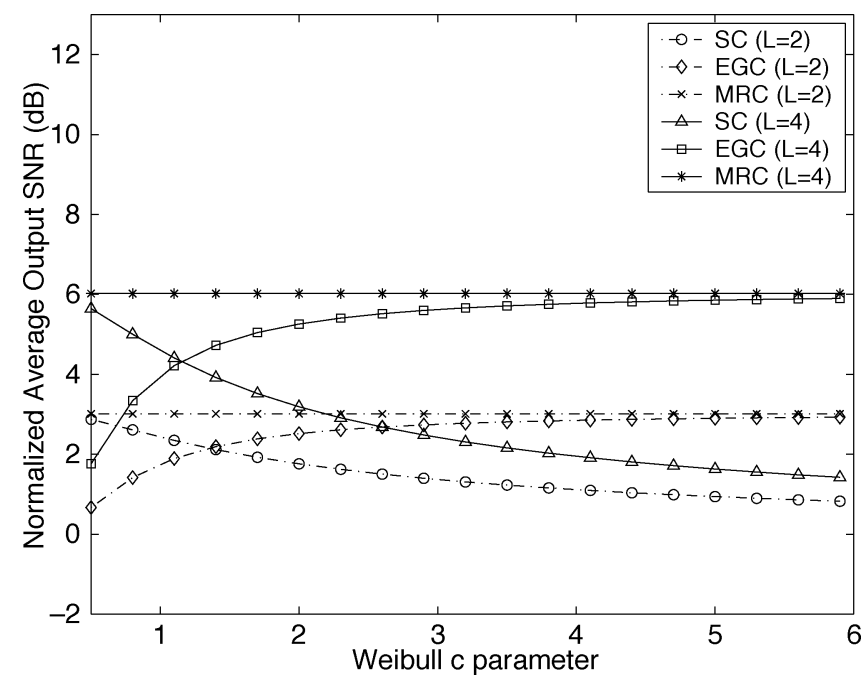

Fig. 1. Average output SNR normalized to the first-branch average output SNR versus the Weibull $c$ parameter for single-channel reception and multichannel diversity reception, with $L=2$ and $L=4$.

\section{Maximal Ratio Combining (MRC)}

The $k$ th moment of the output SNR $Y$ for MRC is

$$
\begin{aligned}
\mathbb{E}\left[Y^{k}\right] & =\mathbb{E}\left[\left(\sum_{i=1}^{L} X_{i}^{2}\right)^{k}\right] \\
& =\sum_{n_{1}+n_{2}+\cdots+n_{L}=k}\left(\begin{array}{c}
k \\
n_{1}, \cdots, n_{L}
\end{array}\right) \prod_{i=1}^{L} \mathbb{E}\left[X_{i}^{2 n_{i}}\right]
\end{aligned}
$$

where $\left(\begin{array}{c}k \\ n_{1}, \cdots, n_{L}\end{array}\right)=k ! /\left(n_{1} ! \cdots n_{L} !\right)$ is the multinomial coefficient. When $k=1$, the first moment of $Y$ gives the average output SNR for MRC as $\overline{\mathrm{SNR}_{\mathrm{o}, \mathrm{mrc}}}=L \mathbb{E}\left[X_{1}^{2}\right]=L \beta^{2 / c} \Gamma(1+$ $2 / c)$.

The OP for MRC diversity reception is given by

$$
P_{\text {out }, \operatorname{mrc}}(\xi)=\operatorname{Pr}(Y<\xi)=\operatorname{Pr}\left(\sum_{i=1}^{L} X_{i}^{2}<\xi\right)
$$

or

$$
P_{\text {out }, \operatorname{mrc}}(\xi)=\frac{1}{2 \pi j} \int_{\sigma-j \infty}^{\sigma+j \infty} \frac{\left[\mathcal{M}_{X}\left(s, \frac{c}{2}\right)\right]^{L}}{s} e^{s \xi} d s
$$

where $j^{2}=-1$ and $\sigma$ is a constant chosen in the region of the convergence of the complex plane.

Calculations of ASERs of MRC for many digital linear modulations on Weibull channels are direct extensions of the MGF method for other fading channels [7, Ch. 9].

\section{NuMERICAL RESULTS AND DISCUSSIONS}

Fig. 1 shows the average output SNR (normalized to the average output SNR of the first branch) versus the Weibull $c$ parameter for single-channel and multichannel diversity reception. The two horizontal lines at 3 and $6 \mathrm{~dB}$ indicate the diversity order of MRC at $L=2$ and $L=4$, respectively. The normalized output SNR for SC approaches $0 \mathrm{~dB}$ when the channel becomes static ( $c$ goes to infinity). This is expected since when

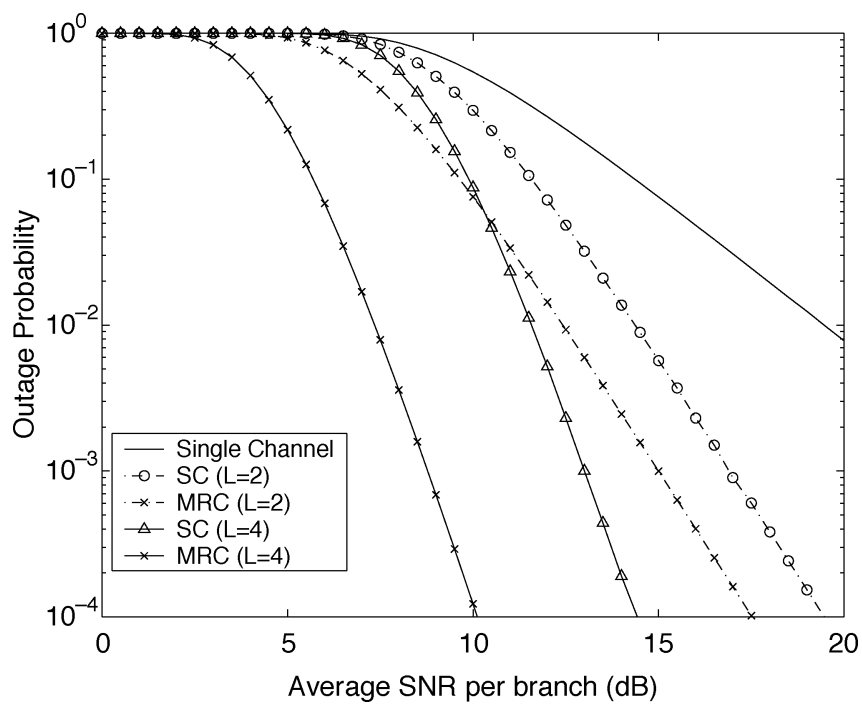

Fig. 2. OP versus average SNR per branch for single-channel reception, SC reception, and $\mathrm{MRC}$ reception at outage threshold $\xi=10 \mathrm{~dB}$ when $c=4$.

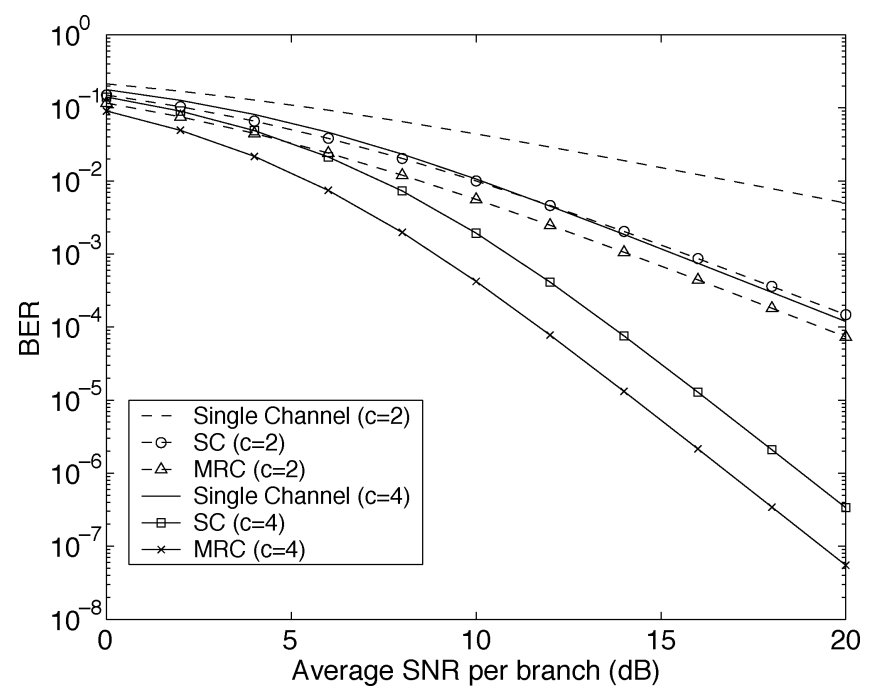

Fig. 3. Average BER versus average SNR per branch for CBFSK with single-channel reception, SC reception $(L=2)$, and MRC reception $(L=2)$ on Weibull fading channels when $c=2$ and $c=4$.

the channel becomes static, the performance of SC will be identical to that of single-channel reception. For comparison purposes, we have also included the normalized output SNR for equal-gain combining (EGC) reception. As expected, when the channel becomes less faded, the SNR performance of EGC approaches that of MRC. It is also of interest to note that the SC can have better SNR performance than EGC when the channel is severely faded, i.e., $c<1$. While this may, perhaps, seem surprising upon first inspection, we note that the discussion in $[12$, Sec. VII $]$ provides useful insight into the possibility of this observation.

Fig. 2 shows the OP versus average branch SNR for singlechannel reception and diversity reception with outage threshold $\xi=10 \mathrm{~dB}$ for diversity orders $L=2$ and $L=4$. As expected, the outage performance improves when the diversity order increases, and the outage performance of MRC reception outperforms that of SC reception with the same level of fading severity. Fig. 3 shows the average BER performance for coherent binary 


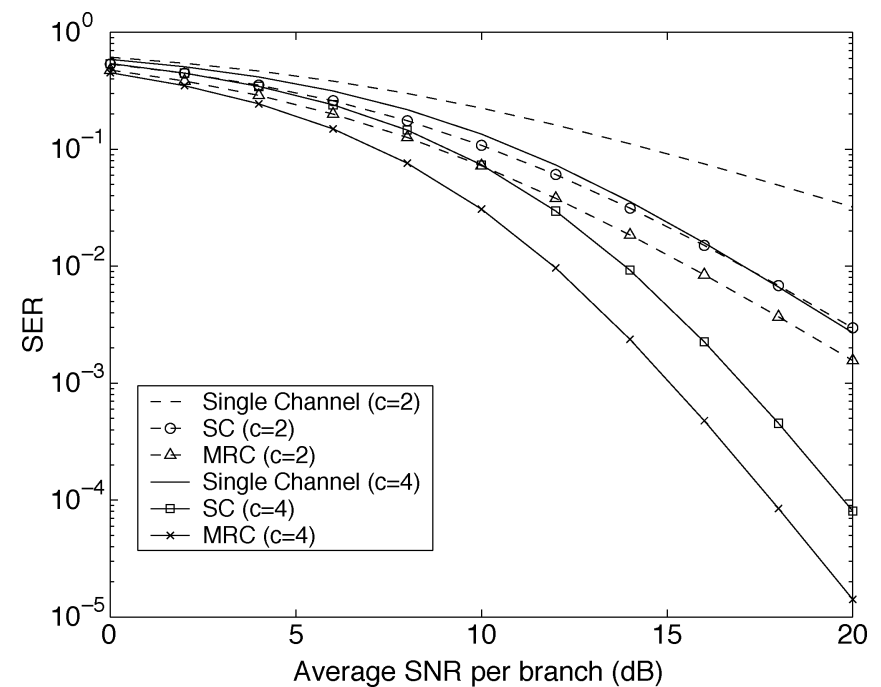

Fig. 4. ASER versus average SNR per branch for 8-PSK with single-channel reception, $\mathrm{SC}$ reception $(L=2)$, and MRC reception $(L=2)$ on Weibull fading channels when $c=2$ and $c=4$.

frequency-shift keying (CBFSK) with single-channel reception and dual-diversity reception. As we expect, the BER performance of diversity reception outperforms single-channel reception, and a lightly faded Weibull fading channel has a better BER performance than another Weibull fading channel with stronger fading. Similar observations can be made for the ASER performance of 8-ary phase-shift keying (PSK) in Fig. 4.

\section{APPENDIX \\ DERIVATION OF (4)}

In this appendix, we derive the integral identity in (4) using a Mellin transform approach. ${ }^{3}$ The Mellin transform of $f(x)$, with respect to the complex parameter $s$, is defined as $M(s)=\int_{0}^{\infty} x^{s-1} f(x) d x$, and the inverse Mellin transform is given by $f(x)=(1 / 2 \pi j) \int_{c-j \infty}^{c+j \infty} M(s) x^{-s} d s$, where $c$ is a suitably chosen number.

Putting $f(x)=e^{-\alpha x^{r}}$, the Mellin transform of $f(x)$ becomes

$$
M(s)=\int_{0}^{\infty} x^{s-1} e^{-\alpha x^{r}} d x=\frac{1}{r} \alpha^{-\frac{s}{r}} \Gamma\left(\frac{s}{r}\right) .
$$

Therefore, from the definition of inverse Mellin transform, we have

$$
\begin{aligned}
e^{-\alpha x^{r}} & =\frac{1}{2 \pi j} \int_{c^{\prime}-j \infty}^{c^{\prime}+j \infty} \frac{1}{r} \alpha^{-\frac{s}{r}} \Gamma\left(\frac{s}{r}\right) x^{-s} d s \\
& =\frac{1}{2 \pi j} \int_{c-j \infty}^{c+j \infty} \alpha^{-s} \Gamma(s) x^{-r s} d s
\end{aligned}
$$

\footnotetext{
${ }^{3} \mathrm{~A}$ reviewer has pointed out an alternative derivation of (4), based on using the relation between Fox's $H$-function [13], [14] and the $G$-function [14], [15].
}

where $c^{\prime}$ and $c$ are two suitably chosen constants. Substituting (21) into (3) and integrating with respect to $x$, we get

$$
I=\frac{1}{2 \pi j} \int_{c-j \infty}^{c+j \infty} \alpha^{-s} z^{r s-p} \Gamma(s) \Gamma(p-r s) d s .
$$

Since $r$ is assumed to be integer, the Gauss multiplication formula [10, eq. (8.335)] allows us to write

$$
\Gamma(p-r s)=(2 \pi)^{\frac{1-r}{2}} r^{p-r s-\frac{1}{2}} \prod_{k=1}^{r} \Gamma\left(\frac{p+k-1}{r}-s\right) .
$$

From (23) and (22), we can write

$$
\begin{aligned}
I=\frac{(2 \pi)^{\frac{1-r}{2}} r^{p-\frac{1}{2}} z^{-p}}{2 \pi j} & \int_{c-j \infty}^{c+j \infty}\left(\frac{z^{r}}{\alpha r^{r}}\right)^{s} \Gamma(s) \\
& \times \prod_{k=1}^{r} \Gamma\left(\frac{p+k-1}{r}-s\right) d s .
\end{aligned}
$$

Now comparing (24) with the definition of Meijer's $G$ function [10, eq. (9.301)], we obtain (4) as desired.

\section{REFERENCES}

[1] N. H. Shepherd, "Radio wave loss deviation and shadow loss at 900 MHz," IEEE Trans. Veh. Technol., vol. VT-26, pp. 309-313, Nov. 1977.

[2] H. Hashemi, "The indoor radio propagation channel," Proc. IEEE, vol. 81, pp. 943-968, July 1993.

[3] G. Tzeremes and C. G. Christodoulou, "Use of Weibull distribution for describing outdoor multipath fading," in Proc. IEEE Antennas, Propagation Soc. Int. Symp., vol. 1, San Antonio, TX, June 2002, pp. 232-235.

[4] "Coverage prediction for mobile radio systems operating in the 800/900 MHz frequency range," IEEE Trans. Veh. Technol., vol. 37, pp. 3-70, Feb. 1988.

[5] M.-S. Alouini and M. K. Simon, "Performance of generalized selection combining over Weibull fading channels," in Proc. IEEE Vehicular Technology Conf., Atlantic City, NJ, Oct. 2001, pp. 1735-1739.

[6] N. C. Sagias, D. A. Zogas, G. K. Karagiannidis, and G. S. Tombras, "Performance analysis of switched diversity receivers in Weibull fading," Electron. Lett., vol. 39, no. 20, pp. 1472-1474, Oct. 2003.

[7] M. K. Simon and M.-S. Alouini, Digital Communication Over Fading Channels: A Unified Approach to Performance Analysis. New York: Wiley, 2000.

[8] A. G. Constantine and N. I. Robinson, "The Weibull renewal function for moderate to large arguments," Computat. Stat. Data Anal., vol. 24, pp. 9-27, 1997.

[9] N. G. Bakhoom, "Asymptotic expansions of the function $F_{x}=$ $\int_{0}^{\infty} e^{-u^{k}+x u} d u$," Proc. London Math. Soc. Ser. 2, vol. 35, pp. 83-100, 1993.

[10] I. S. Gradshteyn and I. M. Ryzhik, Table of Integrals, Series and Products, 6th ed. New York: Academic, 2000.

[11] Y. L. Luke, The Special Functions and Their Approximations. New York: Academic, 1969, vol. I.

[12] D. Brennan, "Linear diversity combining techniques," Proc. IRE, vol. 47, pp. 1075-1102, June 1959.

[13] S. Hegyi, "A powerful generalization of the NBD suggested by Peter Carruthers," in Proc. Int. Workshop Multiparticle Production, Mátraháza, Hungary, 1999, pp. 272-286.

[14] A. M. Mathai and R. K. Saxena, The H-Function With Applications in Statistics and Other Disciplines. New Delhi, India: Wiley, 1978.

[15] G. Huo and M.-S. Alouini, "Another look at the BER performance of $\mathrm{FFH} / \mathrm{BFSK}$ with product combining over partial-band jammed Rayleigh-fading channels," IEEE Trans. Veh. Technol., vol. 50, pp. 1203-1215, Sept. 2001. 